\title{
Identification, Structural Characterization and Gene Expression Analysis of Members of the Nuclear Factor-Y Family in Chickpea (Cicer arietinum L.) under Dehydration and Abscisic Acid Treatments
}

\author{
Ha Duc Chu ${ }^{1}{ }^{\circledR}$, Kien Huu Nguyen ${ }^{1,2,3}$, Yasuko Watanabe ${ }^{3}$, Dung Tien Le ${ }^{1}$, Thu Ly Thi Pham ${ }^{1}$, \\ Keiichi Mochida ${ }^{4,5,6,7}$ and Lam-Son Phan Tran 2,3,*(D) \\ 1 Agricultural Genetics Institute, Vietnam Academy of Agricultural Sciences, Pham Van Dong Road, \\ North Tu Liem District, Hanoi City 122300, Vietnam; hachu_amser@yahoo.com (H.D.C.); \\ kienbio280888@gmail.com (K.H.N.); letiendzung@yahoo.com (D.T.L.); phamthilythu@yahoo.com (T.L.T.P.) \\ 2 Plant Stress Research Group \& Faculty of Applied Sciences, Ton Duc Thang University, \\ Ho Chi Minh City 700000, Vietnam \\ 3 Stress Adaptation Research Unit, RIKEN Center for Sustainable Resource Science, 1-7-22, Suehiro-cho, \\ Tsurumi, Yokohama 230-0045, Japan; yasuko.watanabe@riken.jp \\ 4 Bioproductivity Informatics Research Team, RIKEN Center for Sustainable Resource Science, 1-7-22, \\ Suehiro-cho, Tsurumi, Yokohama 230-0045, Japan; keiichi.mochida@riken.jp \\ 5 Microalgae Production Control Technology Laboratory, RIKEN Baton Zone Program, \\ RIKEN Cluster for Science, Technology and Innovation Hub, 1-7-22 Suehiro-cho, Tsurumi-ku, Yokohama, \\ Kanagawa 230-0045, Japan \\ 6 Institute of Plant Science and Resources, Okayama University, 2-20-1 Chuo, Kurashiki, \\ Okayama 710-0046, Japan \\ 7 Kihara Institute for Biological Research, Yokohama City University, 641-12 Maioka-cho, Totsuka-ku, \\ Yokohama, Kanagawa 244-0813, Japan \\ * Correspondence: sontran@tdtu.edu.vn or son.tran@riken.jp; Tel.: +81-45-503-9593; Fax: +81-45-503-9591
}

Received: 6 September 2018; Accepted: 18 October 2018; Published: 23 October 2018

\begin{abstract}
In plants, the Nuclear Factor-Y (NF-Y) transcription factors (TFs), which include three distinct types of NF-YA, NF-YB, and NF-YC TFs, have been identified to play key roles in the regulation of various plant growth and developmental processes under both normal and environmental stress conditions. In this work, a total of 40 CaNF-Y-encoding genes, including eight CaNF-YAs, 21 CaNF-YBs, and 11 CaNF-YCs, were identified in chickpea, and their major gene and protein characteristics were subsequently obtained using various web-based tools. Of our interest, a phylogenetically-based analysis predicted 18 CaNF-Ys (eight CaNF-YAs, seven CaNF-YBs, and three CaNF-YCs) that potentially play roles in chickpea responses to dehydration according to their close relationship with the well-characterized GmNF-Ys in soybean. These results were in good agreement with the enrichment of drought-responsive cis-regulatory motifs and expression patterns obtained from in silico analyses using publically available transcriptome data. Most of the phylogenetically predicted drought-responsive CaNF- $Y$ genes ( 15 of 18 ) were quantitatively validated to significantly respond to dehydration treatment in leaves and/or roots, further supporting the results of in silico analyses. Among these CaNF-Y genes, the transcript levels of CaNF-YA01 and CaNF-YC10 were the most highly accumulated in leaves (by approximately eight-fold) and roots (by approximately 18-fold), respectively, by dehydration. Furthermore, 12 of the 18 CaNF-Y genes were found to be responsive to the most well-known stress hormone, namely abscisic acid (ABA), in leaves and/or roots, suggesting that these genes may act in chickpea response to dehydration in ABA-dependent manner. Taken together, our study has provided a comprehensive and fundamental information for further functional analyses of selected $C a N F-Y$ candidate genes, ultimately leading to the improvement of chickpea growth under water-limited conditions.
\end{abstract}


Keywords: abscisic acid; Cicer arietinum; dehydration; expression analysis; Nuclear Factor-Y; phylogenetic analyses; quantitative PCR; transcription factor

\section{Introduction}

Chickpea (Cicer arietinum L.) has been identified as a native leguminous crop of the Mediterranean region, especially in the Afro-Asian countries [1]. The crop is highly valued as its seeds provide an excellent source of high-quality proteins, carbohydrates, minerals, and vitamins [2,3]. Thus, chickpea has been considered an economically healthy food and valuable feed for humans and livestock, respectively $[4,5]$. In addition, cultivation of chickpea plants can improve soil nitrogen level via symbiotic nitrogen fixation [6]. Since chickpea is mainly grown under rain-fed conditions, water shortage is one of the major factors that greatly limits its growth and productivity [7].

To develop strategies for development of improved drought-tolerant chickpea, a great effort has been made to identify and characterize drought-responsible genes, particularly those encoding transcription factors (TFs), from this legume species [8-10]. The Nuclear Factor-Y (NF-Y) TFs, which compose of NF-YA, NF-YB and NF-YC subclass TFs, have been shown to be involved in regulation of many developmental processes and responses to various environmental stimuli [11-14]. With the great advances in the 'omics' era, members of the NF-Y TF family have been identified and characterized in several plant species, including monocotic species, such as common wheat (Triticum aestivum) [15], rice (Oryza sativa) [16,17], Brachypodium distachyon [18], barley (Hordeum vulgare) [19], foxtail millet (Setaria italica) [20], maize (Zea mays) [21,22] and sorghum (Sorghum bicolor) [23], as well as dicotic species, such as Arabidopsis thaliana [24,25], common bean (Phaseolus vulgaris) [26], canola (Brassica napus) [27,28], soybean (Glycine max) [29], grape (Vitis vinifera) [30], tomato (Solanum lycopersicum) [31], upland cotton (Gossypium hirsutum) [32], sweet orange (Citrus sinensis) [33], and castor bean (Ricinus communis) [34]. However, the NF-Y TFs have not been systematically identified and characterized yet at a genome-wide level in the economically important chickpea, even though its genome sequence has been publically available to the research community for several years $[35,36]$.

In the present study, we have made an effort to identify all putative members (referred to as CaNF-Y TFs) of the NF-Y family in chickpea and provide comprehensive information on their structural characteristics. A list of putative dehydration-responsive CaNF-Y genes was proposed using phylogenetic analysis. Screening of the hormone- and/or stress-related cis-acting motifs in the putative promoter sequences of the identified CaNF-Y genes was then performed to increase the confidence of the phylogenetic analysis-based prediction. To gain a first glance into their functions, we analyzed the transcript patterns of the CaNF- $Y$ genes in various major organs using available RNA-seq resources. Finally, to precisely identify the CaNF-Y genes with potential functions in regulation of chickpea responses to dehydration, quantitative PCR (qPCR) was used to separately examine the expression of the identified CaNF- $Y$ genes in roots and leaves of chickpea plants exposed to dehydration or abscisic acid (ABA) treatment.

\section{Results and Discussion}

\subsection{Identification and Annotation of the CaNF-Y Genes in Chickpea}

To obtain all potential genes encoding CaNF-Y TFs, an intensive search was conducted in the two major TF databases, namely the iTAK (Plant Transcription factors \& Protein Kinase Identifier and Classifier) [37] and the PlantTFDB (Plant Transcription Factor Database) [38], which predicted the CaNF-Y TFs in the 'kabuli' chickpea [35]. As a result, a total of 40 putative CaNF-Y genes, classified into three groups, CaNF-YAs (eight members), CaNF-YBs (21 members), and CaNF-YCs (11 members), were identified in the chickpea genome ('kabuli' type) (Table 1; Dataset S1). The general annotations of each of the identified CaNF-Y TFs were then obtained from the genome assembly 
(GCF_000331145.1) [35] and provided in Table 1. Next, the multiple alignments of the full-length CaNF-YAs, CaNF-YBs and CaNF-YCs were separately constructed using the Clustal X software [39] (Figure S1). The core sequences of the CaNF-YA TFs contain two distinct domains, the 'NF-YB and NF-YC Interaction' domain ( 20 amino-acid residues) and the 'DNA Binding' domain ( 20 amino-acid residues), separated by an $\sim 10$-amino-acid linker region (Figure S1A). The conserved core sequences of the CaNF-YB TFs possess two functional domains involved in the heterodimer formation ('NF-YA Interaction' and 'NF-YC Interaction' domains), and a domain required for DNA interaction in the heterotrimer complex ('DNA Binding' domain) (Figure S1B). The CaNF-YC conserved core regions consist of three domains, namely two short 'NF-YA Interaction' domains involved in the heterodimer interaction with the CaNF-YAs, and a region called 'NF-YB Interaction' involved in dimerization with the CaNF-YBs (Figure S1C).

To define the structural features of the CaNF- $Y$ genes in chickpea, the exon/intron organization of the CaNF-Y genes was investigated by alignments of their coding DNA sequences (CDSs) and related genomic sequences using the GSDS (Gene Structure Display Server) tool [40]. All eight CaNF-YA genes shared similar structure, with five exons and four introns (six genes) or six exons and five introns (two genes), while the number of exons of CaNF-YB genes varies from one to six (Table 1). Seven of the 11 CaNF-YC genes are intronless, while CaNF-YCO1 and CaNF-YC09 have 2/1 and 4/3 exons/introns, respectively, and both CaNF-YC07 and CaNF-YC11 consist of six exons and five introns (Table 1). In comparison with other plant species, multiple exon/intron gene organization was observed not only in the NF-YA genes of chickpea (Table 1), but also in the NF-YA genes of B. napus [27], P. vulgaris [26] and S. lycopersicum [31], suggesting that this is a representative feature of the NF-YA genes, at least in plants. In addition, the intronless phenomenon is common in the NF- YB and NF-YC genes in various plant species, including chickpea (Table 1), S. bicolor (seven of 11 SbNF-YBs and 10 of 14 SbNF-YCs are intronless) [23], C. sinensis (six of 11 CsNF-YBs and three of five CsNF-YCs are intronless) [33] and R. communis (eight of 12 RcNF-YBs and three of seven TcNF-YCs intronless) [34]. It has been well-established that introns are known to be the essential entities of eukaryotic gene structure with numerous functions, such as in exon shuffling [41], alteration of the gene expression [42], and regulation of the evolutionary rate of genes [43]. Thus, our results indicated that the introns may have some critical roles in the evolution and functions of the NF-Y gene family in chickpea, and perhaps in other plant species as well.

We next obtained detailed information on the typical characteristics of all the identified CaNF-Y TFs, which included protein length, molecular weight $(\mathrm{mW})$, instability index, theoretical isoelectric point (pI) and grand average of hydropathicity (GRAVY), by subjecting their full-length protein sequence to the ExPASy ProtParam web-based tool for an analysis [44]. Our data indicated that the CaNF-YAs possess the protein length between 206 to 339 amino-acid residues, while the sizes of the CaNF-YBs and CaNF-YCs were ranged from 104 to 244 and 114 to 357 amino-acid residues, respectively (Table 1). The mWs of CaNF-Ys were recorded from 11.71 to $40.52 \mathrm{kDa}$, and their pI values varied from the acidic $(\mathrm{pI}=4.42)$ to base $(\mathrm{pI}=9.17)$ (Table 1$)$. A majority of the CaNF-Ys, excluding CaNF-YB01, CaNF-YB07, CaNF-YB09, CaNF-YB11, CaNF-YB18, CaNF-YB19, and CaNF-YB20, showed an instability index higher than 40 (Table 1); and thus, these CaNF-Ys could be classified as unstable proteins [45]. Additionally, the GRAVY values of almost all CaNF-Ys, except CaNF-YC10, were recognized to be negative (Table 1), revealing that these CaNF-Ys are hydrophilic [46]. 
Table 1. 40 CaNF-Y (Nuclear Factor-Y) genes identified in the chickpea genome and their general characteristics. Data were obtained from ${ }^{1}$ PlantTFDB, ${ }^{2}$ NCBI, ${ }^{3}$ iTAK, ${ }^{4}$ ExPASy ProtParam, and ${ }^{5}$ GSDS. pI, isoelectric point; $\mathrm{mW}$, molecular weight (kDa); II, instability index; GRAVY, grand average of hydropathy; ' - ', no information.

\begin{tabular}{|c|c|c|c|c|c|c|c|c|c|}
\hline Gene Name & Protein ID ${ }^{1}$ & Locus ID $^{1,2}$ & 'Ca' Code ${ }^{3}$ & Protein Size ${ }^{4}$ & $\mathrm{pI}^{4}$ & $\mathrm{~mW}^{4}$ & II $^{4}$ & GRAVY $^{4}$ & Number of Exons \\
\hline CaNF-YA01 & XP_012571402 & LOC101492587 & Ca_02713 & 339 & 8.45 & 37.64 & 54.73 & -0.69 & 5 \\
\hline CaNF-YA02 & XP_004486451 & LOC101496019 & Ca_07995 & 206 & 6.79 & 22.76 & 61.06 & -0.96 & 5 \\
\hline CaNF-YA03 & XP_004494259 & LOC101506076 & Ca_00866 & 333 & 9.43 & 36.16 & 43.44 & -0.50 & 5 \\
\hline CaNF-YA04 & XP_004497643 & LOC101500532 & Ca_14455 & 335 & 9.17 & 36.84 & 60.91 & -0.56 & 5 \\
\hline CaNF-YA05 & XP_004500196 & LOC101504175 & Ca_15574 & 337 & 6.22 & 36.54 & 59.60 & -1.08 & 5 \\
\hline CaNF-YA06 & XP_004510428 & LOC101496153 & Ca_10039 & 313 & 8.70 & 34.32 & 56.63 & -0.60 & 6 \\
\hline CaNF-YA07 & XP_004510989 & LOC101500168 & Ca_20250 & 332 & 9.41 & 37.40 & 59.43 & -0.84 & 6 \\
\hline CaNF-YA08 & XP_012574919 & LOC101488265 & Ca_11593 & 244 & 9.86 & 26.93 & 56.04 & -0.65 & 5 \\
\hline CaNF-YB01 & XP_004486346 & LOC101490518 & Ca_07906 & 157 & 6.43 & 17.42 & 38.92 & -0.59 & 1 \\
\hline CaNF-YBO2 & XP_004488253 & LOC101497461 & Ca_20300 & 130 & 5.93 & 14.77 & 40.39 & -0.75 & 1 \\
\hline CaNF-YBO3 & XP_004488351 & LOC 101507640 & Ca_22468 & 156 & 4.63 & 17.40 & 49.85 & -0.48 & 5 \\
\hline CaNF-YB04 & XP_004491514 & LOC101513559 & Ca_09721 & 194 & 6.21 & 20.99 & 45.91 & -0.67 & 1 \\
\hline CaNF-YB05 & XP_004491660 & LOC101504444 & Ca_09724 & 201 & 4.79 & 22.74 & 52.76 & -0.52 & 2 \\
\hline CaNF-YB06 & XP_004493942 & LOC101512494 & Ca_12013 & 171 & 6.10 & 18.76 & 42.49 & -0.82 & 5 \\
\hline CaNF-YBO7 & XP_004498435 & LOC101491567 & Ca_13093 & 147 & 6.51 & 16.06 & 34.12 & -0.57 & 2 \\
\hline CaNF-YB08 & XP_004498102 & LOC101490166 & Ca_20009 & 181 & 4.94 & 20.48 & 50.79 & -0.64 & 1 \\
\hline CaNF-YBO9 & XP_004498386 & LOC101500751 & Ca_13089 & 137 & 6.96 & 15.24 & 29.99 & -0.56 & 2 \\
\hline CaNF-YB10 & XP_004495979 & LOC101499868 & Ca_03440 & 142 & 5.39 & 16.49 & 58.00 & -1.07 & 1 \\
\hline CaNF-YB11 & XP_004496024 & LOC101514675 & - & 104 & 7.64 & 11.71 & 20.95 & -0.69 & 3 \\
\hline CaNF-YB12 & XP_004495242 & LOC101489398 & Ca_07860 & 174 & 6.09 & 19.22 & 55.44 & -0.82 & 5 \\
\hline CaNF-YB13 & XP_004503068 & LOC101504826 & - & 184 & 5.04 & 21.01 & 51.90 & -0.84 & 1 \\
\hline CaNF-YB14 & XP_004504028 & LOC101492000 & Ca_09605 & 190 & 5.80 & 20.96 & 46.23 & -0.66 & 1 \\
\hline CaNF-YB15 & XP_004503919 & LOC101512746 & - & 223 & 6.21 & 25.87 & 45.74 & -1.12 & 1 \\
\hline CaNF-YB16 & XP_004508609 & LOC101491613 & Ca_06841 & 146 & 5.31 & 16.73 & 49.21 & -0.91 & 1 \\
\hline CaNF-YB17 & XP_004508593 & LOC101512449 & Ca_06596 & 219 & 5.39 & 24.33 & 46.12 & -0.32 & 6 \\
\hline CaNF-YB18 & XP_004507593 & LOC101497005 & Ca_20238 & 136 & 6.17 & 15.31 & 30.16 & -0.62 & 1 \\
\hline CaNF-YB19 & XP_004507590 & LOC101496026 & - & 244 & 6.49 & 27.34 & 34.09 & -0.89 & 2 \\
\hline CaNF-YB20 & XP_004516103 & LOC101498601 & Ca_20798 & 191 & 6.21 & 20.47 & 37.65 & -0.72 & 1 \\
\hline CaNF-YB21 & XP_004516113 & LOC101501902 & Ca_20790 & 227 & 4.42 & 24.83 & 49.49 & -0.53 & 2 \\
\hline CaNF-YC01 & XP_004488773 & LOC101507861 & Ca_13620 & 357 & 7.31 & 40.52 & 60.84 & -0.71 & 2 \\
\hline CaNF-YCO2 & XP_004487742 & LOC101503561 & Ca_06933 & 114 & 8.64 & 12.60 & 57.68 & -0.12 & 1 \\
\hline CaNF-YCO3 & XP_004486310 & LOC101502053 & Ca_00637 & 223 & 5.24 & 25.76 & 62.49 & -0.74 & 1 \\
\hline CaNF-YCO4 & XP_004494629 & LOC101508013 & Ca_01175 & 260 & 6.03 & 28.95 & 59.81 & -0.61 & 1 \\
\hline CaNF-YC05 & XP_004495741 & LOC101507067 & Ca_03698 & 256 & 5.78 & 28.52 & 67.12 & -0.65 & 1 \\
\hline
\end{tabular}


Table 1. Cont

\begin{tabular}{|c|c|c|c|c|c|c|c|c|c|}
\hline Gene Name & Protein ID ${ }^{1}$ & Locus ID ${ }^{1,2}$ & 'Ca' Code ${ }^{3}$ & Protein Size ${ }^{4}$ & $\mathrm{pI}^{4}$ & $\mathrm{~mW}^{4}$ & II $^{4}$ & GRAVY $^{4}$ & Number of Exons 5 \\
\hline CaNF-YC06 & XP_004501704 & LOC101502982 & Ca_01433 & 213 & 5.03 & 23.84 & 56.43 & -0.52 & 1 \\
\hline CaNF-YCO7 & XP_004499778 & LOC101492987 & Ca_17100 & 295 & 5.11 & 32.73 & 50.38 & -0.88 & 6 \\
\hline CaNF-YC08 & XP_012571846 & LOC101509194 & Ca_11344 & 150 & 5.88 & 17.20 & 75.58 & -0.61 & 1 \\
\hline CaNF-YC09 & XP_004505981 & LOC101509858 & Ca_14578 & 219 & 9.63 & 25.06 & 46.15 & -1.11 & 4 \\
\hline CaNF-YC10 & XP_004507577 & LOC101491701 & - & 124 & 9.15 & 13.57 & 57.66 & 0.08 & 1 \\
\hline CaNF-YC11 & XP_004511091 & LOC101513228 & Ca_20191 & 309 & 5.21 & 33.96 & 46.02 & -0.88 & 6 \\
\hline
\end{tabular}




\subsection{Chromosomal Localization and Prediction of the Duplication Events of CaNF-Y Genes}

Out of the 40 identified CaNF-Ys, a total of 37 genes, except CaNF-YA08, CaNF-YB20 and CaNF-YB21, could be mapped on the chickpea genome consisting of eight chromosomes. An interesting feature of the chromosomal localization of the CaNF-Y genes is that chromosomes I and IV share the highest number of CaNF-Y genes (eight genes), whereas chromosome VIII does not contain even a single CaNF- $Y$ gene (Figure 1). Although NF- $Y$ genes have been identified in various eukaryotic organisms, their number varies from a few in animal and yeast [47] to many in plant species (Table S1). For example, the number of genes encoding NF-Y TFs in plant species varies from 22 (six NF-YA, $11 N F-Y B$, and five NF-YC genes in C. sinensis) [33] to 68 (21 NF-YA, $32 N F-Y B$, and $15 N F-Y C$ genes in G. max) [29] (Table S1). This relatively high variation appears to be a common feature in the plant kingdom, as it might help plants form a flexible, versatile regulatory system to accommodate complex and diverse environmental conditions [14].

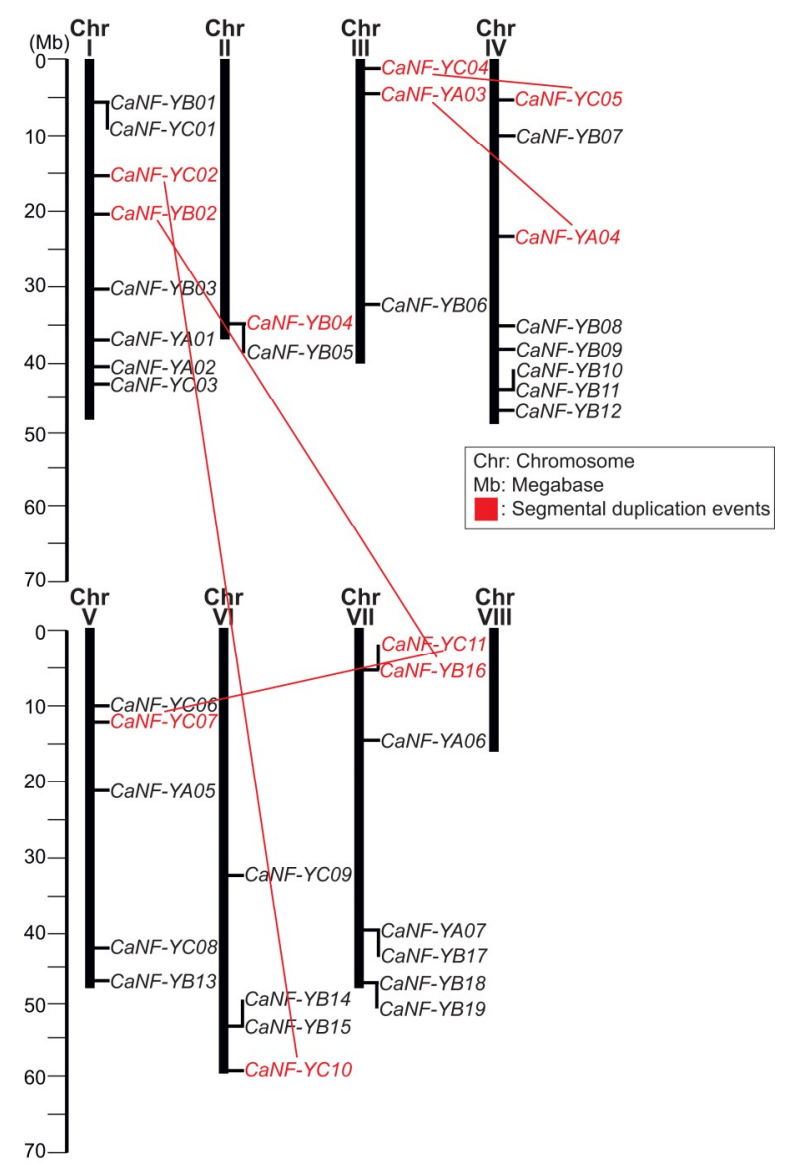

Figure 1. Chromosomal localization of 40 identified CaNF-Y genes in the chickpea genome. Red color indicates the CaNF- $Y$ genes that form duplicated pairs with the criterion of higher than $70 \%$ nucleotide identity. Three CaNF-Y genes, CaNF-YA08, CaNF-YB20, and CaNF-YB21 could not be assigned to any chromosomes in the currently available chickpea genome version.

To evaluate the molecular evolution of the CaNF- $Y$ genes, we assessed the duplication rates among the identified CaNF-Y genes using the preset criterion $>70 \%$ identity at the nucleotide level. By using the sequence identity matrix function in BioEdit software [48], out of $40 \mathrm{CaNF-} Y$ genes, six duplicated pairs were identified that shared higher identity than 70.3\% nucleotide homology (CaNF-YC07 and CaNF-YC11) to 79.6\% (CaNF-YC04 and CaNF-YC05) (Table 2). Five of these, except the pair formed by CaNF-YB04 located on chromosome II and CaNF-YB20 on the unplaced scaffolds, were recorded on different chromosomes, whereas no tandem duplication events were found (Figure 1). This finding suggested that these five duplicated pairs were the results of segmental duplication events, which 
might contribute to the expansion of the CaNF-Y gene family in chickpea as both tandem and segmental duplication events have been considered to play essential roles in expansion and maintenance of gene families in genomes [49]. In previous studies, six segmental (of 12 genes) and four tandem (of nine genes) duplicated events (the identity of the genes $\geq 80 \%$ was used as the criterion in analysis) were identified among 39 members of the SiNF-Y gene family in S. italica [20], while two tandem and six

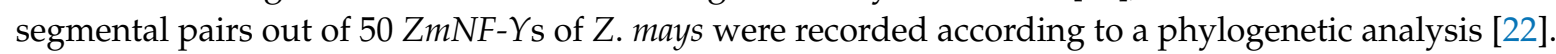
More recently, seven groups of 15 OsNF-Y genes from rice were produced from segmental duplication blocks, while four OsNF-Y genes (two pairs) could be assigned to tandem duplication events [17] according to the Rice Genome Annotation Project [50]. Furthermore, out of eight duplicated pairs of 16 SbNF-Ys in S. bicolor, no tandem duplication event was found [23]. These results together suggested that the expansion of the NF-Y gene families in plant kingdom might be due to segmental duplication events rather than tandem duplication events.

Table 2. Prediction of duplication events among the identified CaNF-Y genes in chickpea. US, unplaced scaffolds; Ka, value indicating nonsynonymous substitutions per nonsynonymous site; Ks, value indicating synonymous substitutions per synonymous site; $\mathrm{T}$, approximate time of the duplication event; Mya, million years ago.

\begin{tabular}{cccccccc}
\hline Gene Pairs & $\begin{array}{c}\text { Chromosome } \\
\text { Localization }\end{array}$ & $\begin{array}{c}\text { Duplication } \\
\text { Event }\end{array}$ & $\begin{array}{c}\text { Identity } \\
\text { Level (\%) }\end{array}$ & Ka & Ks & Ka/Ks & T (Mya) \\
\hline CaNF-YA03/CaNF-YA04 & Chr III/Chr IV & Segmental & 76.8 & 0.26 & 0.21 & 1.24 & 16.15 \\
CaNF-YB04/CaNF-YB20 & Chr II/US & Unknown & 72.3 & 0.16 & 0.68 & 0.23 & 52.31 \\
CaNF-YB02/CaNF-YB16 & Chr I/Chr VII & Segmental & 72.3 & 0.13 & 0.73 & 0.18 & 56.15 \\
CaNF-YC02/CaNF-YC10 & Chr I/ Chr VI & Segmental & 76.8 & 0.19 & 0.13 & 1.46 & 10.00 \\
CaNF-YC04/CaNF-YC05 & Chr III/Chr IV & Segmental & 79.6 & 0.20 & 0.31 & 0.64 & 23.85 \\
CaNF-YC07/CaNF-YC11 & Chr V/Chr VII & Segmental & 70.3 & 0.29 & 0.33 & 0.88 & 25.38 \\
\hline
\end{tabular}

In the next line of our study, we predicted the pressure of natural selection on the expansion of the CaNF-Y genes during evolution process by calculating the ratio of nonsynonymous (Ka) and synonymous (Ks) nucleotide substitution rates (Ka/Ks) using the DnaSP software [51]. The Ka/Ks ratios of two duplicated pairs, 'CaNF-YA03 and CaNF-YA04' and 'CaNF-YBO2 and CaNF-YB10', were higher than 1, indicating positive selective pressure [52] (Table 2). On the other hand, the $\mathrm{Ka} / \mathrm{Ks}$ ratios of the other four duplicated pairs, 'CaNF-YB04 and CaNF-YB20', 'CaNF-YB02 and CaNF-YB16', 'CaNF-YCO4 and CaNF-YCO5', and 'CaNF-YCO7 and CaNF-YC11', were lower than 1, revealing the possibility of negative selective pressure associated with the conserved protein sequences [52] (Table 2). Furthermore, the determinations of $\mathrm{Ka}$ and $\mathrm{Ks}$ values allowed us to predict that the segmental duplication events of the CaNF-Y genes might have originated sometimes between 10 million years ago $(\mathrm{Mya})(\mathrm{Ks}=0.13)$ and 56.15 Mya $(\mathrm{Ks}=0.73)($ Table 2$)$.

\subsection{Phylogenetic Analysis-Based Prediction of the CaNF-Y Genes with Drought-Related Functions}

To predict potential functions of the CaNF-Y TFs using phylogenetic analysis, which has been shown to be a reliable method for functional prediction, particularly those involved in plant response to water deficit [8,53], we built an unrooted phylogenetic tree from the full-length protein sequences of all 40 CaNF-Ys and 26 GmNF-Ys (out of 68) of soybean that have been shown to be potentially involved in soybean response to drought [29] (Figure 2). Specifically, 12 GmNF-YA (out of 21), 10 GmNF-YB (out of 32), and four GmNF-YC (out of 15) TFs, whose encoding genes were characterized to be drought-responsive in soybean, were included in this phylogenetic analysis-based functional prediction [29] (Figure 2). The principle of this method is that if the CaNF-Ys were phylogenetically classified into the same clades with the GmNF-Ys, their encoding genes might also be drought-responsive [54], i.e., these CaNF-Ys might play a role in chickpea response to drought as their partners in soybean [29]. We found that all eight CaNF-YAs, and seven out of 21 CaNF-YBs were grouped in the same clusters with 12 GmNF-YAs and $10 \mathrm{GmNF-YBs,} \mathrm{respectively} \mathrm{(Figure} \mathrm{2A,B).}$ 
Among 11 CaNF-YCs, three members, namely CaNF-YC02, CaNF-YC04, and CaNF-YC10, were arranged into two groups with four GmNF-YCs (Figure 2C). These results suggest that these orthologous CaNF-Y-encoding genes might have a drought-responsive expression profile, and potentially play a role in chickpea adaptation to drought. For instance, GmNF-YA11 was noted to be induced in soybean leaves by drought [29], suggesting that its ortholog CaNF-YA07 might also be up-regulated in chickpea leaves by water deficit, and both GmNF-YA11 and CaNF-YA07 might function in improving leaves-associated trait(s) for better drought adaptation (Figure 2A). On the other hand, GmNF-YB02 was up-regulated in soybean roots by drought [29], implying that its closest neighbor, CaNF-YB04, would also be induced in chickpea roots by water stress, and both two orthologous genes might regulate roots-related traits to enhance drought tolerance (Figure 2B). It is also worth mentioning that the drought-responsive GmNF-YA01 gene was highly expressed in nodules [29], suggesting that their orthologous chickpea partner CaNF-YA04 might have an important role associated with nodule functioning (Figure 2A,B). These candidate genes from chickpea and soybean could be selected for further in-depth studies for improvement of nitrogen-fixing efficiency that has been well-known to be adversely affected by water stress [55-57].

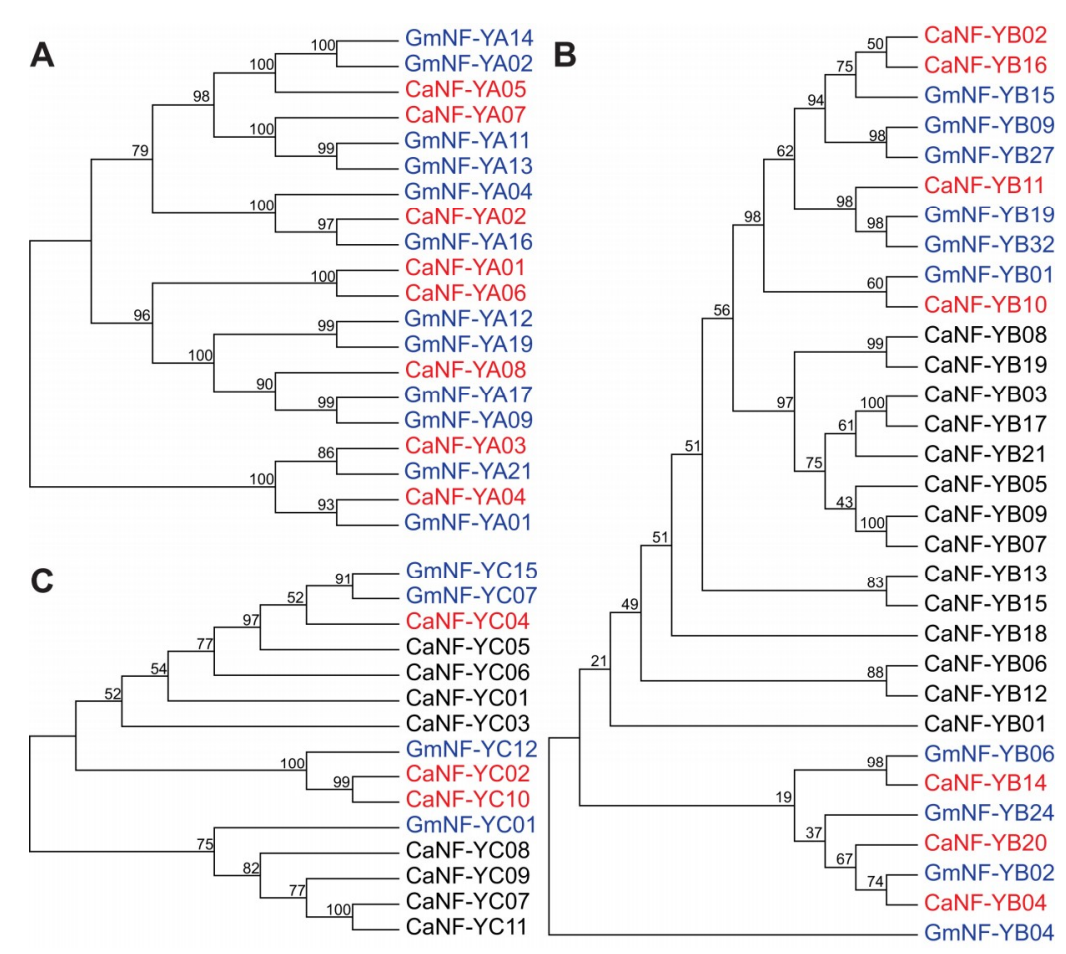

Figure 2. Phylogenetic analysis-based prediction of drought-responsive CaNF-Y genes. The unrooted phylogenetic trees were built from the full-length (A) CaNF-YAs, (B) CaNF-YBs and (C) CaNF-YCs, and their respective well-characterized GmNF-YA, GmNF-YB and GmNF-YC proteins using MEGA7. The GmNF-Ys were indicated by blue color, while the predicted drought-related CaNF-Ys were highlighted by red color. Bootstrap values $>50 \%$ are showed next to the branch.

\subsection{Prediction of the Cis-Acting Motifs in the Promoter Region of CaNF-Y Genes}

Another approach that can be considered for functional prediction of the genes of interest is in silico identification of the well-established cis-acting motifs in the promoter sequences of the genes $[58,59]$. To reveal the potential roles of CaNF-Y genes in the plant adaptation to environmental stresses, with particular interest in drought, 11 well-known stress-related and eight well-known hormone-related cis-regulatory elements were used in a search for their presence(s) in the 1000-bp promoter regions of all identified $40 \mathrm{CaNF}-\mathrm{Y}$ genes (Table S2). For instance, ABRE (ABA-responsive element) was included as it was found to be important for signal transduction of ABA, an essential 
phytohormone for plant environmental stress adaptation [60]. DRE (dehydration-responsive element), MBS (MYB-binding site), MYCR (MYC-binding site), CE3 (coupling element 3), T/G Box, EE (evening element) and NACR (binding site of NAC TFs) were used in this in silico analysis as they were characterized as dehydration-inducible cis-regulatory elements [61-64] (Table S2), of which DRE and NACR are famous for their roles in mediating the drought-inducible functions of DREB and NAC TFs in many plants species [64-67], including legumes [68-70]. Furthermore, HSE (heat stress element), which has been considered as a heat-responsive cis-motif, and ICEr2 (induction of CBF expression region 2) and LTRE (low temperature-responsive element), which have been recognized as cold-responsive cis-elements $[63,64]$, were also included in our analysis (Table S2). As a result, the promoters of most CaNF-Y genes (37 of 40), excluding CaNF-YB07, CaNF-YB11, and CaNF-YB14, contained at least one type of stress-related cis-motifs (Table S2). The EE and HSE elements were enriched in the promoter regions of 16 and 18 CaNF- $Y$ genes, respectively, whereas CE3 and LTRE showed their presences in the promoters of only two CaNF-Y genes (Table S2). Interestingly, we found that a majority of phylogenetically predicted CaNF-Y genes (14 of 18), except CaNF-YA07, CaNF-YB10, CaNF-YB11 and CaNF-YB14, contained at least one dehydration-responsive cis-element (Figure 2; Table S2). Among them, the promoters of CaNF-YA04 and CaNF-YC10 shared the highest accumulation of dehydration-responsive cis-motifs, with four and three motifs, respectively (Table S2). As for hormone-related cis-motifs, we found that ABRE was distributed in the promoters of eight CaNF-Y genes (Table S2), among which there were five phylogenetically predicted drought-related genes, namely CaNF-YA01, CaNF-YA04, CaNF-YB10, CaNF-YC04, and CaNF-YC10 (Figure 2; Table S2), suggesting that these CaNF-Y genes might be implied in regulation of plant response to drought/dehydration through ABA-dependent pathway. Additionally, analysis of the putative promoter sequences of the 40 CaNF- $Y$ genes showed the presences of cis-motifs related to the jasmonate acid responsiveness (21 of 40), and of cis-elements associated with the gibberellin responsiveness (14 of 40) (Table S2). ERE, an ethylene-responsive element, was detected in the promoter of CaNF-YA01 gene only, while TGA-element involved in auxin responsiveness was found in the promoters of four CaNF-Y genes, namely CaNF-YA01, CaNF-YA04, CaNF-YB06, and CaNF-YB09 (Table S2). TCA-element, a cis-motif involved in salicylic acid responsiveness, was detected in the promoter regions of $10 \mathrm{CaNF-Y}$ genes (Table S2). Taken together, results of our promoter analysis have provided a first glance into the roles of the identified CaNF-Y genes in the regulation of plant stress responses and hormone responsiveness and supported the results of the phylogenetic analysis-based functional prediction of the CaNF-Y genes.

\subsection{Transcript Patterns of the CaNF-Y Genes in Major Organs of Chickpea Plants during Growth and Development}

Investigations of the tissue-specific transcript patterns of CaNF- $Y$ genes in tissues and organs undergoing growth and development can provide helpful insights into their potential functions at various growth stages of plants [71]. In this study, the Chickpea Transcriptome Database (CTDB) [72] was assessed to obtain the transcript patterns of the identified CaNF-Y genes in five major organs, including roots, shoots, mature leaves, flower buds, and young pods [73]. The shoots and roots were sampled from 15-day-old chickpea seedlings grown in culture room [74], while the mature leaves, flower buds, and young pods were harvested from field-grown chickpea plants [73]. The transcriptome atlas reported expression information for a total of only 17 CaNF-Y genes, including four (out of eight) CaNF-YAs, nine (out of 21) CaNF-YBs, and four (out of 11) CaNF-YCs (Figure 3A), while it did not provide any expression data, represented by sequence reads, for the remaining $23 \mathrm{CaNF-Y}$ genes [72]. In general, these 17 CaNF- $Y$ genes showed highly variable expression patterns in the examined organs, and most of them displayed higher transcript levels in flower buds than in other examined organs (Figure 3A). Among them, CaNF-YB03 and CaNF-YC11 were recognized to specifically express in flower buds (Figure 3A), suggesting that they might have a function associated with flower development or flowering time, as reported earlier for several NF-Y genes in A. thaliana $[25,75]$ and 
rice [76]. Additionally, some of the CaNF-Ys, such as CaNF-YB04, CaNF-YB06, and CaNF-YC07, showed differential expression patterns, as they expressed highly in a few particular organs, while lowly in others (Figure 3A). It is worth noting that CaNF-YB20 expressed highly in all five examined organs, while CaNF-YB10 and CaNF-YB14 showed low expression levels in all tissues examined (Figure 3A). Several genes like CaNF-YA03, CaNF-YB17, and CaNF-YB20 appeared to ubiquitously express in all five tissues, suggesting their involvement in controlling general cellular machinery like regulating transcription of 'housekeeping' genes [18]. Alternatively, they may be post-translationally and tissue-specifically modified or may interact with other tissue-specific TFs to induce a particular transcriptional regulation [77].

Next, the root transcriptome data of the 50-day-old (early reproductive stage) chickpea plants obtained during drought stress were used to retrieve the transcript levels of the predicted CaNF-Y genes [9]. Out of $40 \mathrm{CaNF}-Y_{\mathrm{s}}$, expression of 26 genes, including 13 (out of 18) phylogenetically predicted genes, could be detected (Figures 2 and 3B). Eight out of 13 phylogenetically predicted CaNF-Y genes were significantly responsive to drought in the roots (Figures 2 and $3 \mathrm{~B}$ ). More specifically, CaNF-YA01, CaNF-YA04, CaNF-YA05, CaNF-YA06, CaNF-YB04, CaNF-YB10, and CaNF-YC04 were noted to be highly induced in roots, whereas CaNF-YA08 was found to be reduced in roots by drought treatment (Figure 3B). These results collectively indicate the involvement of many CaNF-Y genes in chickpea response to drought.

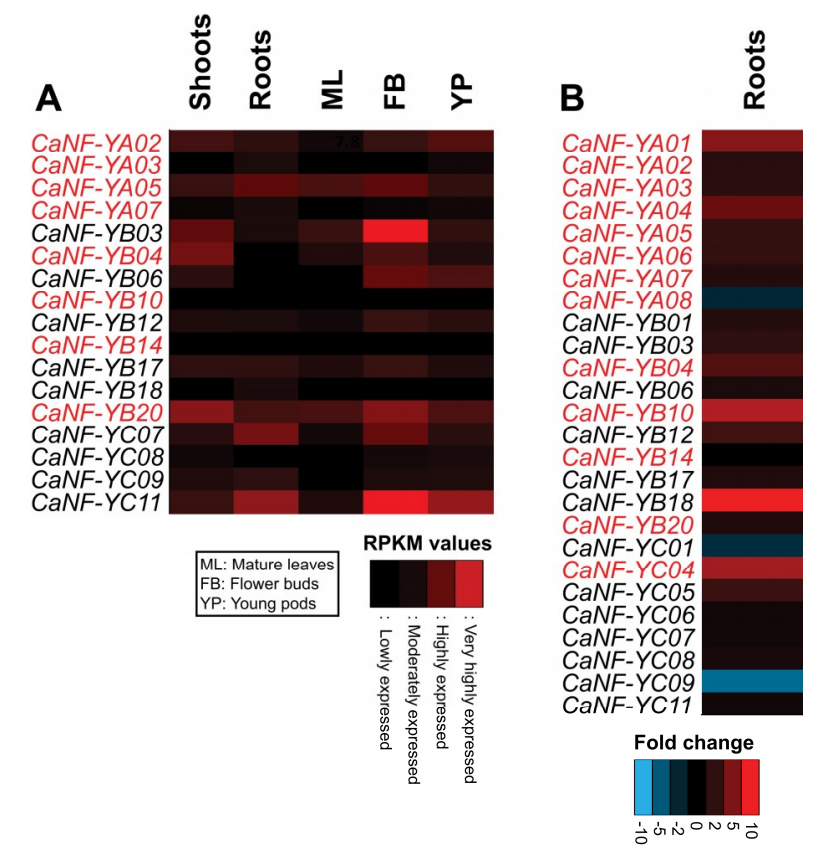

Figure 3. Expression patterns of the CaNF- $Y$ genes in different tissues under normal and drought conditions. (A) Heatmap of expression levels of the CaNF-Y genes in shoots, roots, mature leaves (ML), flower buds (FB) and young pods (YP). Roots and shoots were sampled from 15-day-old chickpea seedlings, while the ML, FB and YP were obtained from chickpea plants grown under field conditions. The reads per kilobase per million (RPKM) with values greater than 3 smaller than/equal 10, greater than 10 smaller than/equal 50, greater than 50 smaller than/equal 100, and greater than 100 are defined as lowly, moderately, highly, and very highly expressed, respectively, as indicated by the colored scale. (B) Heatmap of fold changes in transcript levels of the CaNF-Y genes in chickpea roots under drought. The colored scale represents the fold change of a gene according to the transcriptome analysis. Gene name in red indicates the phylogenetically predicted drought-related CaNF-Y genes. 
2.6. Quantification of Transcript Levels of the Predicted Stress-Related CaNF-Y Genes in Roots and Leaves of Chickpea Plants Exposed to Dehydration or ABA Treatment

Previously, we predicted a total of 18 water stress-related CaNF- $Y$ genes (eight CaNF-YAs, seven CaNF-YBs, and three CaNF-YAs) using a phylogenetic analysis. In this step, as a means to verify their potential role in chickpea response to water deficit, we quantified the transcript levels of these $18 \mathrm{CaNF}-Y$ genes in leaves and roots of chickpea seedlings under dehydration. Results of qPCR indicated that a majority of the 18 predicted CaNF- $Y$ genes (15 of 18) showed significantly altered expression by at least two-fold $(p<0.05)$ in dehydrated roots and/or leaves (Figure 4; Table S4). Eight and five CaNF-Y genes were induced and repressed by at least two-fold in leaf and/or root organs, respectively, under dehydration (Figure 4; Table S4). More specifically, three CaNF- $Y$ genes (CaNF-YA05, CaNF-YA06 and CaNF-YA07) and four CaNF-Y genes (CaNF-YB02, CaNF-YB16, CaNF-YCO2 and CaNF-YC10) were induced in dehydration-treated leaf and root samples, respectively, while CaNF-YA01 was up-regulated in both leaves and roots of chickpea plants subjected to dehydration (Figure 4; Table S4). Furthermore, CaNF-YA01 and CaNF-YC10 was noted to be the most significantly up-regulated gene in stressed leaves (by eight-fold) and roots (by 18-fold), respectively (Figure 4; Table S4). On the other hand, CaNF-YA03 and CaNF-YB20 were repressed in only leaves, whereas CaNF-YA02 and CaNF-YB14 were down-regulated specifically in roots under dehydration (Figure 4; Table S4). CaNF-YA08 gene was recorded to be repressed in both leaves and roots, while CaNF-YB04 was interestingly found to be repressed in leaves but induced in roots during dehydration (Figure 4; Table S4). Interestingly, we found that the expression of CaNF-YA04 in leaves was repressed after $2 \mathrm{~h}$ but induced after $5 \mathrm{~h}$ of dehydration treatment (Figure 4, Table S4). Previous studies have reported the roles of a number of NF-Y genes in the improvements of drought tolerance in various plant species. For instance, overexpression of drought-inducible AtNF-YB1 in Arabidopsis and its orthologous drought-inducible $Z m N F-Y B 2$ in maize were found to enhance drought tolerance of transgenic plants [78]. Likewise, transgenic Arabidopsis plants individually overexpressing osmotic (polyethylene glycol) stress- and ABA-inducible NF-YB genes (e.g., PwNF-YB3 from Picea wilsonii and $P d N F-Y B 7$ from poplar) $[79,80]$, and transgenic rice plants overexpressing a dehydration-induced bermudagrass $N F-Y C$ gene (e.g., Cdt-NF-YC1) conferred improved tolerance under drought [81]. These results, indeed, give an encouragement for detailed functional analyses of selected CaNF-Y genes with the aim to create improved drought-resistant chickpea cultivars. 


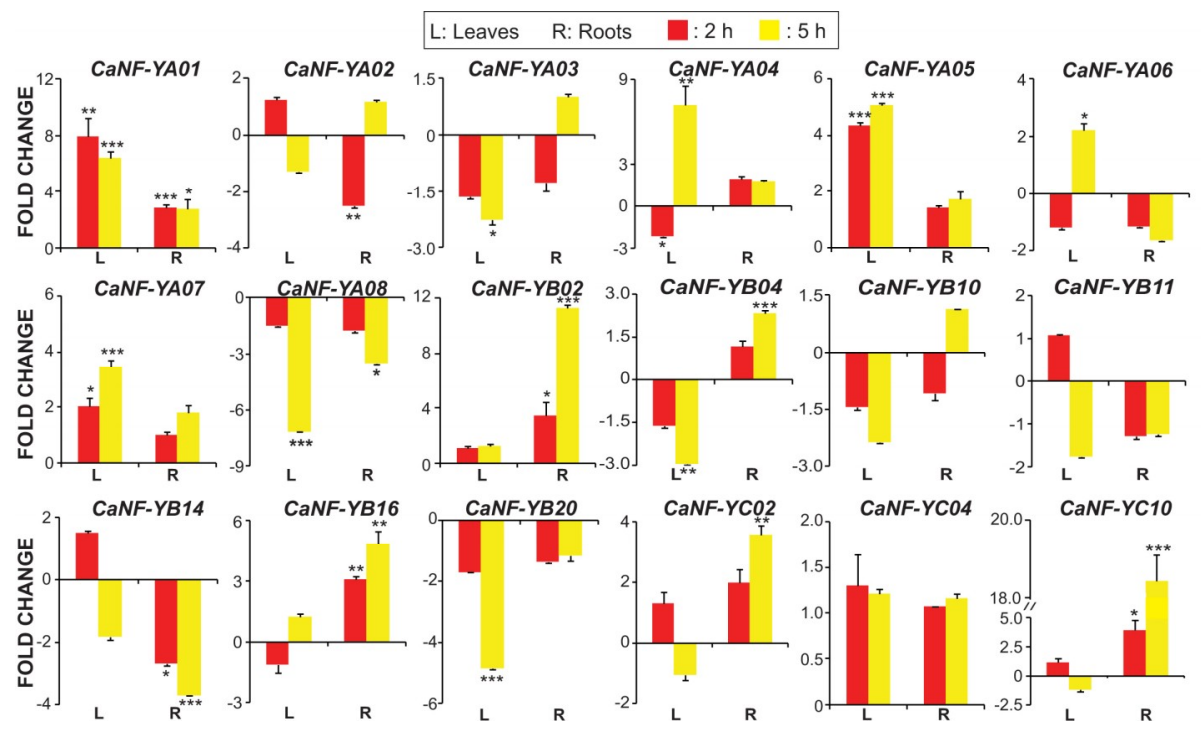

Figure 4. Quantitative expression analysis of the 18 phylogenetically predicted CaNF-Y genes in leaves and roots of chickpea plants subjected to dehydration. Red and yellow columns indicate the fold changes of the CaNF-Y genes after 2 and $5 \mathrm{~h}$ of dehydration treatment, respectively. Error bars indicate the standard errors of three biological replicates $(n=3)$. Statistically significant differences (Student's $t$-test) are shown by asterisks with ${ }^{*} p<0.05,{ }^{* *} p<0.01$ and ${ }^{* *} p<0.001$.

It has been well accepted that $\mathrm{ABA}$ is a major mediator of water-deficit stress adaptation in plants $[60,82,83]$, and genes can act either in ABA-dependent or ABA-independent manner to regulate plant responses to drought/dehydration [84]. In order to identify which CaNF-Y genes act dependently or independently of ABA in chickpea responses to water deficit, we examined the expression of all 18 phylogenetically predicted $C a N F-Y$ genes in ABA-treated chickpea leaves and roots using qPCR. Our results indicated that a total of 12 (out of 18) CaNF-Y genes exhibited expression changes by at least two-fold $(p<0.05)$ in leaves and/or roots of chickpea plants subjected to exogenous ABA treatment (Figure 5; Table S4). In more details, nine and two CaNF-Y genes were induced and repressed in ABA-treated leaves and/or roots, respectively, while only one gene, namely CaNF-YA06, was found to be up-regulated in leaves but down-regulated in roots of ABA-treated plants (Figure 5; Table S4). Furthermore, CaNF-YA06 (by seven-fold) and CaNF-YB16 (by 50-fold) was the most highly up-regulated gene in leaves and roots, respectively, during ABA treatment (Figure 5; Table S4). Out of 15 dehydration-responsive CaNF-Y genes, 11 genes were also responsive to ABA in leaves and/or leaves (Figure 5; Table S4), suggesting that a majority of CaNF-Y genes can act in the ABA-dependent fashion to regulation chickpea responses to water scarcity. CaNF-YB10 was not responsive to the dehydration treatment in both leaves and roots (Figure 4; Table S4) but was induced in roots by ABA (Figure 5; Table S4). It is worth mentioning that CaNF-YA01 was the most highly up-regulated gene under both dehydration (in leaves by eight-fold) and ABA treatments (in roots by $\sim$ nine-fold) (Figure 5; Table S4). 

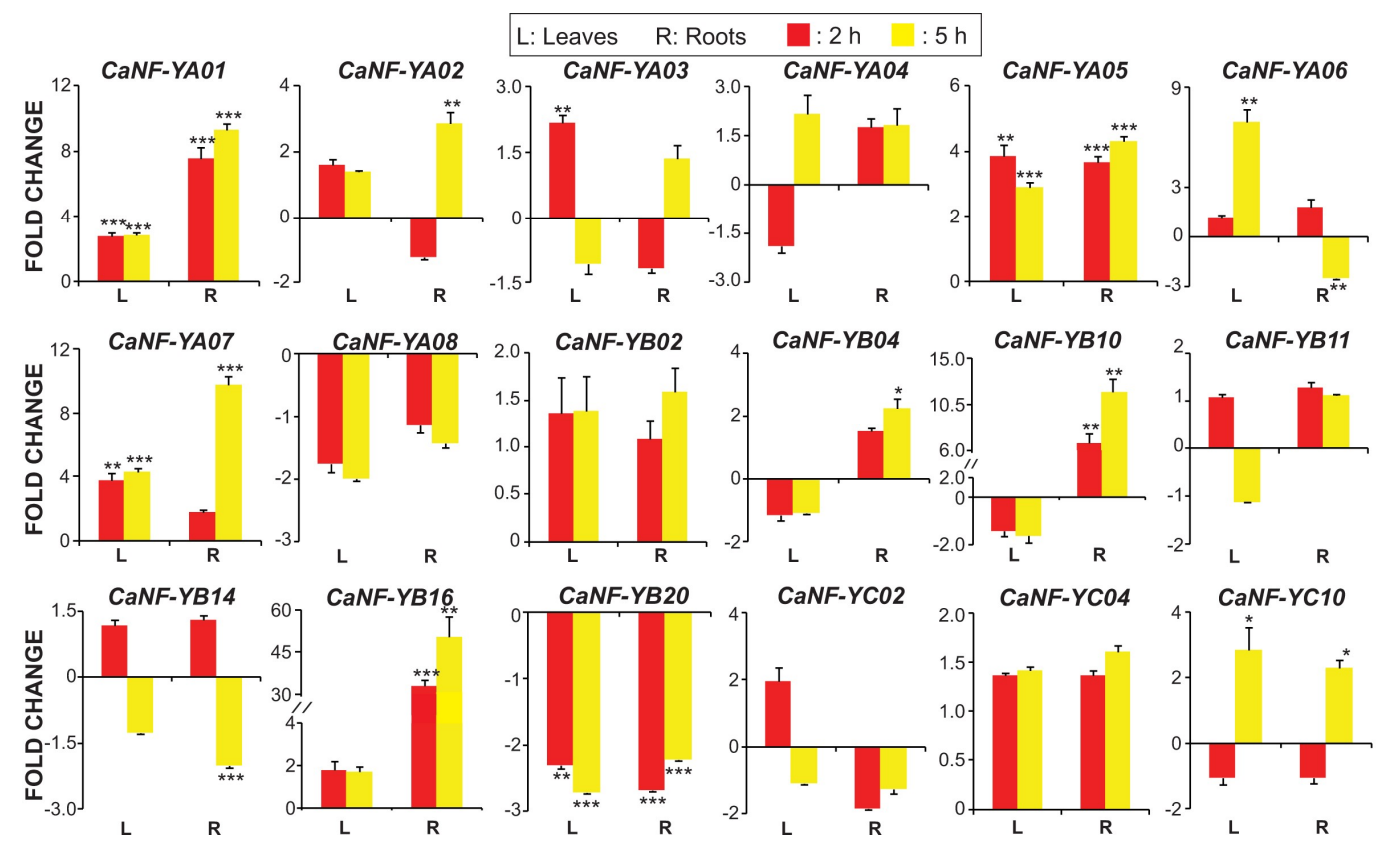

Figure 5. Quantitative expression analysis of the 18 phylogenetically predicted CaNF-Y genes in leaves and roots of chickpea plants subjected to abscisic acid (ABA) treatment. Red and yellow columns indicate the fold changes of the CaNF-Y genes after 2 and $5 \mathrm{~h}$ of ABA treatment, respectively. Error bars indicate the standard errors of three biological replicates $(n=3)$. Statistically significant differences (Student's $t$-test) are shown by asterisks with ${ }^{*} p<0.05,{ }^{* *} p<0.01$, and ${ }^{* * *} p<0.001$.

\section{Materials and Methods}

\subsection{Identification and Annotation of the CaNF-Y Genes}

The iTAK database (Plant Transcription factor \& Protein Kinase Identifier and Classifier, http://itak.feilab.net/cgi-bin/itak/index.cgi) [37] and the PlantTFDB (Plant Transcription Factor Database, http:/ / planttfdb.cbi.pku.edu.cn/) [38] TF databases were used to collect the amino acid sequences, coding DNA sequences (CDSs), and the protein identifiers of all potential CaNF-Y TFs. The protein sequence of each CaNF-Y was then searched against the current 'kabuli' genome assembly (GCF_000331145.1) available in NCBI RefSeq [35] to obtain the general gene annotation features, including gene identifier, locus identifier, genomic DNA sequence, and chromosomal distribution.

\subsection{Gene Duplication Analysis of the CaNF-Ys}

Gene duplication events of the CaNF-Y genes were analyzed based on the identity of their CDSs. First, the CDSs of CaNF-Y genes were aligned in the Clustal X (v. 2.1) software [39]. The identity matrix between the CDSs of CaNF-Y genes was then constructed in the BioEdit (v. 7.2.5) [48] and exported into Microsoft Excel. A pair of duplicated genes was defined as sharing $>70 \%$ identity at the nucleotide level. Particularly, the segmental duplication events were predicted based on the locations of the duplicated pair on distinct chromosomes. Duplicated genes closely linked within $20 \mathrm{~kb}$ of each other on a chromosome were considered as tandem duplicated genes [20].

To predict the pressure of natural selection on the expansion of the CaNF- $Y$ genes during evolution, values of non-synonymous substitutions per non-synonymous site $(\mathrm{Ka})$, and values of synonymous substitutions per synonymous site (Ks) of duplicated genes were calculated using the DnaSP (v. 5.0) software [51]. The Ka/Ks ratio between paralogs was analyzed to predict the mode of selection. The $\mathrm{Ka} / \mathrm{Ks}$ value $>1$ indicate positive selective pressure and assume the divergent protein sequences, whereas a ratio $<1$ indicates the possibility of negative selective pressure associated with conserved 
protein sequences [85]. The approximate time of the duplication events was predicted as previously studied and expressed in 'million years ago' (Mya) [23].

\subsection{Protein Features and Gene Organization of the CaNF-Ys}

The physical and chemical characteristics of CaNF-Y TFs, including the protein length (amino acid), molecular weight $(\mathrm{mW}, \mathrm{kDa})$, theoretical isoelectric point $(\mathrm{pI})$, instability index, and grand average of hydropathicity (GRAVY), were calculated using the ExPASy ProtParam (https:/ / www.web.expasy.org/protparam) [44]. The exon/intron organization of the CaNF-Y genes was defined by subjecting the CDS and genomic sequences to an analysis using the GSDS (v. 2.0) (Gene Structure Display Server, http:/ /gsds.cbi.pku.edu.cn/) [40].

\subsection{Conserved Domains and Phylogenetic Analysis-Based Prediction of the CaNF-Y Proteins}

The full-length protein sequences of CaNF-YA, CaNF-YB and CaNF-YC TFs were used in separate alignments to determine the conserved domains of each NF-Y subfamily using the Clustal X (v. 2.1) software [39]. Gap extension penalty of 0.2 and gap open penalty of 10 were applied in the analysis [39]. The alignments of the conserved domains of separate NF-Y subunits were exported into the BioEdit software [48] for obtaining a graphical view. To predict the CaNF-Y TFs with drought-related function, a Neighbor-Joining unrooted tree was created from the full-length CaNF-Y TFs and the published drought-related NF-Y proteins of soybean [29] using the MEGA (v. 7.0) software [86] with the preset parameters of 1000 bootstrap replications. The cut-off value of $50 \%$ was used for the condensed tree.

\subsection{Prediction of the Stress- and Hormone-Related Cis-Regulatory Elements}

The 1000-bp promoter regions of CaNF-Y genes were extracted from the chickpea genome sequence [35]. Eight hormone-related cis-regulatory elements, including GARE-motif (-AAACAGAor -TCTGTTG-) and P-box (-GCCTTTTGAGT-) involved in gibberellin responsiveness, TGA-element (-AACGAC-) involved in auxin responsiveness, ERE (ethylene-responsive element, -ATTTCAAA-) involved in ethylene responsiveness, TCA-element (-CAGAAAAGGA- or -GAGAAGAATA-) involved in salicylic acid responsiveness, TGACG-motif (-TGACG-) and CGTCA-motif (-CGTCA-) involved in jasmonic acid responsiveness, and ABRE (ABA-responsive element, -CACGTG- or -TACGTG-) involved in ABA responsiveness [60], were used for a search of the promoter sequences of all the CaNF-Y genes using the PlantCARE (Plant Cis-Acting Regulatory Element, http:// bioinformatics.psb.ugent.be/webtools/plantcare/html/) [87] (Table S2). A total of 11 stress-related cis-regulatory elements, including HSE (heat stress element, -AAAAAATTTC-) involved in heat stress responsiveness, LTRE (low temperature-responsive element, -CCGAAA-) and ICEr2 (Induction of CBF expression region 2, -ACTCCG-) involved in low temperature responsiveness, TC-rich repeats (-GTTTTCTTAC- or -ATTTTCTTCA-) involved in defense and stress responsiveness [63,64], and seven drought/dehydration-responsive cis-motifs were also subjected to a search in the promoter sequences of the CaNF-Y genes (Table S2) using either PlantCARE [87] or manually. The seven cis-motifs well-established as drought/dehydration-responsive were as follows: DRE (dehydration-responsive element, -GCCGAC- or -ACCGAC-), CE3 (coupling element 3, -CACGCG-), T/G Box (-CACGTT-), EE (evening element, -AATATC-) [61,62], MBS (MYB-binding site, -CAACTG- or -TAACTG-) [62], MYCR (MYC-binding site, -CACATG-), and NACR (binding site of drought-inducible NAC TFs, -CACGCA-) $[61,63,64]$ (Table S2).

\subsection{Transcript Patterns of the CaNF-Y Genes in Different Organs}

Transcript patterns of the CaNF- $Y$ genes in five major organs were determined by using the expression data obtained from the CTDB (http://www.nipgr.res.in/ctdb.html) [72], which were obtained from 454 pyrosequencing [73]. The root and shoot samples were harvested from 15-day-old seedlings grown in pots, filled with vermiculite and agropeat (1:1), in culture room [74], while the flower bud, mature leaf and young pod samples were obtained from the plants grown under field 
conditions [73]. To determine the expression patterns of the CaNF- $Y$ genes in chickpea roots under drought stress, the publically available transcriptome data obtained from roots of the desi 'ICC 4958' chickpea plants treated with drought was also used (GSE70274) [9]. In their study, the 'ICC 4958' plants were grown and subjected to drought stress using a 'dry-down' method [9]. The roots of the chickpea plants in their early reproductive stage (approximately 50-day-old) were collected when the available soil water fraction (ASWF) reached 0.2, while the pots of control plants were maintained at optimum water level, with the ASWF of 0.9 [9].

\subsection{Growth, and Dehydration and ABA Treatments of Chickpea Plants}

Growth of chickpea (Hashem 'kabuli' cultivar) plants in pots containing vermiculite was followed as essentially described in Ha et al. [8]. Well-watered, ABA $(100 \mu \mathrm{M})$, and dehydration treatments of vermiculite-grown 9-day-old chickpea seedlings for 2 and $5 \mathrm{~h}$ were carried out as previously reported [8]. Relative water contents of $55 \%$ and $33 \%$ were noted in plant samples after 2 and $5 \mathrm{~h}$ of dehydration treatment, respectively.

\subsection{Expression Analysis of CaNF-Y Genes by qPCR}

Leaf and root samples were separately collected for RNA extraction, which was conducted using RNAeasy Plant Mini Kit and QIAcube system as described by the provided protocol (Qiagen). RNA quantification, DNaseI treatment and cDNA synthesis were performed following previously published methods $[8,88]$. The specific forward and reverse primers for each CaNF- $Y$ gene were designed using the Primer 3 [89] (Table S3). Quantitative PCR (qPCR) was carried out using MX300 as previously reported [8]. The reference gene used in data analysis was Initiation factor 4a (IF4a) [74]. The Student's $t$-test was used to assess the statistically significant differences in transcript levels between treatments. A gene was defined as ABA- and/or dehydration-responsive gene, if its expression level was changed by at least two-fold $\left({ }^{*} p<0.05,{ }^{* *} p<0.01\right.$ and $\left.{ }^{* * *} p<0.001\right)$ under ABA and/or dehydration treatment(s).

\section{Conclusions}

In the present study, a total of 40 CaNF-Y genes, including eight CaNF-YAs, 21 CaNF-YBs and 11 CaNF-YCs, were identified in the chickpea genome, and their major characteristics were provided. Using various in silico analysis approaches, including phylogenetic analysis, occurrences of well-known hormone- and stress-responsive cis-regulatory motifs, as well as data mining and analysis of available expression data, we have provided the first glance into the potential functions of the identified CaNF-Y genes. Furthermore, using qPCR, we revealed that the phylogenetic analysis-based prediction is a reliable method, which showed that a majority of the predicted genes (15 of 18 genes) are dehydration-responsive; and thus, potentially are involved in chickpea responses to water deficit. Additionally, results of qPCR also indicated that 12 of 18 predicted genes act in an ABA-dependent manner to regulate chickpea adaptation to water scarcity. CaNF-YA01 and CaNF-YC10 were the most highly up-regulated genes under dehydration, with approximately eight- and 18-fold increase in transcript level in dehydrated leaves and roots, respectively. In addition, CaNF-YA01 was found as the most highly up-regulated gene under both dehydration (in leaves) and ABA (in roots) treatments. Taken together, our study has provided the basic features of the CaNF-Y TFs in chickpea and characterized the responses of the CaNF-Y genes to water-deficit stress.

Supplementary Materials: Supplementary materials can be found at http:/ / www.mdpi.com/1422-0067/19/ 11/3290/s1. Dataset S1: Nucleotide and amino acid sequences of 40 CaNF-Y genes, Figure S1: The multiple alignments of full-length (A) CaNF-YA (B) CaNF-YB and (C) CaNF-YC TFs as determined using Clustal X (v. 2.1) software. Conserved domains were indicated by thick black lines above the sequences. The conserved residues were marked in white color in the black background, Table S1: Summary of the members of NF-Y genes in plant species, Table S2: Stress- and hormone-related cis-regulatory elements in the promoter regions of 40 CaNF-Y genes, Table S3: List of primers for quantitative expression of predicted CaNF- $Y$ genes, Table S4: Summary of expression profiles of 18 predicted $C a N F-Y$ genes under dehydration and ABA treatment in leaves and/or roots. 
Author Contributions: L.-S.P.T. designed the study. H.D.C., D.T.L., K.H.N., Y.W., T.L.T.P. and K.M. performed the experiments. H.D.C. and K.H.N. analyzed the data. H.D.C. and L.-S.P.T. wrote the manuscript. All the authors revised and approved the manuscript.

Funding: This research was partly funded by the International Program Associate of RIKEN (Japan), and the National Foundation for Science \& Technology Development of Vietnam (Grant No. 106-NN.02-2013.46).

Conflicts of Interest: The authors declare no conflict of interest.

\section{References}

1. Ohri, D.; Pal, M. The origin of chickpea (Cicer arietinum L.): Karyotype and nuclear DNA amount. Heredity 1991, 66, 367-372. [CrossRef]

2. Rachwa-Rosiak, D.; Nebesny, E.; Budryn, G. Chickpeas-Composition, nutritional value, health benefits, application to bread and snacks: A review. Crit. Rev. Food Sci. Nutr. 2013, 55, 1137-1145. [CrossRef] [PubMed]

3. Acharjee, S.; Sarmah, B.K. Biotechnologically generating 'super chickpea' for food and nutritional security. Plant Sci. 2013, 207, 108-116. [CrossRef] [PubMed]

4. Bird, L.G.; Pilkington, C.L.; Saputra, A.; Serventi, L. Products of chickpea processing as texture improvers in gluten-free bread. Food Sci. Technol. Int. 2017, 23, 690-698. [CrossRef] [PubMed]

5. El-Beltagi, H.S.; El-Senousi, N.A.; Ali, Z.A.; Omran, A.A. The impact of using chickpea flour and dried carp fish powder on pizza quality. PLoS ONE 2017, 12, e0183657. [CrossRef] [PubMed]

6. Esfahani, M.N.; Sulieman, S.; Schulze, J.; Yamaguchi-Shinozaki, K.; Shinozaki, K.; Tran, L.S. Approaches for enhancement of $\mathrm{N}_{2}$ fixation efficiency of chickpea (Cicer arietinum L.) under limiting nitrogen conditions. Plant Biotechnol. J. 2014, 12, 387-397. [CrossRef] [PubMed]

7. Khodadadi, M. Effect of drought stress on yield and water relative content in chickpea. Int. J. Agron. Plant Prod. 2013, 4, 1168-1172.

8. Ha, C.V.; Esfahani, M.N.; Watanabe, Y.; Tran, U.T.; Sulieman, S.; Mochida, K.; Nguyen, D.V.; Tran, L.S. Genome-wide identification and expression analysis of the CaNAC family members in chickpea during development, dehydration and ABA treatments. PLoS ONE 2014, 9, e114107. [CrossRef] [PubMed]

9. Garg, R.; Shankar, R.; Thakkar, B.; Kudapa, H.; Krishnamurthy, L.; Mantri, N.; Varshney, R.K.; Bhatia, S.; Jain, M. Transcriptome analyses reveal genotype- and developmental stage-specific molecular responses to drought and salinity stresses in chickpea. Sci. Rep. 2016, 6, 19228. [CrossRef] [PubMed]

10. Mahdavi Mashaki, K.; Garg, V.; Nasrollahnezhad Ghomi, A.A.; Kudapa, H.; Chitikineni, A.; Zaynali Nezhad, K.; Yamchi, A.; Soltanloo, H.; Varshney, R.K.; Thudi, M. RNA-Seq analysis revealed genes associated with drought stress response in kabuli chickpea (Cicer arietinum L.). PLoS ONE 2018, 13, e0199774. [CrossRef] [PubMed]

11. Myers, Z.A.; Holt, B.F., 3rd. NUCLEAR FACTOR-Y: Still complex after all these years? Curr. Opin. Plant Biol. 2018, 45, 96-102. [CrossRef] [PubMed]

12. Zanetti, M.E.; Ripodas, C.; Niebel, A. Plant NF-Y transcription factors: Key players in plant-microbe interactions, root development and adaptation to stress. Biochim. Biophys. Acta 2017, 1860, 645-654. [CrossRef] [PubMed]

13. Samad, A.F.A.; Sajad, M.; Nazaruddin, N.; Fauzi, I.A.; Murad, A.M.A.; Zainal, Z.; Ismail, I. Microrna and transcription factor: Key players in plant regulatory network. Front. Plant Sci. 2017, 8, 565. [CrossRef] [PubMed]

14. Petroni, K.; Kumimoto, R.W.; Gnesutta, N.; Calvenzani, V.; Fornari, M.; Tonelli, C.; Holt, B.F., 3rd.; Mantovani, R. The promiscuous life of plant NUCLEAR FACTOR Y transcription factors. Plant Cell 2012, 24, 4777-4792. [CrossRef] [PubMed]

15. Stephenson, T.J.; McIntyre, C.L.; Collet, C.; Xue, G.P. Genome-wide identification and expression analysis of the NF-Y family of transcription factors in Triticum aestivum. Plant Mol. Biol. 2007, 65, 77-92. [CrossRef] [PubMed]

16. Thirumurugan, T.; Ito, Y.; Kubo, T.; Serizawa, A.; Kurata, N. Identification, characterization and interaction of HAP family genes in rice. Mol. Genet. Genom. 2008, 279, 279-289. [CrossRef] [PubMed]

17. Yang, W.; Lu, Z.; Xiong, Y.; Yao, J. Genome-wide identification and co-expression network analysis of the OsNF-Y gene family in rice. Crop J. 2017, 5, 21-31. [CrossRef] 
18. Cao, S.; Kumimoto, R.W.; Siriwardana, C.L.; Risinger, J.R.; Holt, B.F., 3rd. Identification and characterization of NF-Y transcription factor families in the monocot model plant Brachypodium distachyon. PLoS ONE 2011, 6, e21805. [CrossRef] [PubMed]

19. Liang, M.; Hole, D.; Wu, J.; Blake, T.; Wu, Y. Expression and functional analysis of NUCLEAR FACTOR-Y, subunit B genes in barley. Planta 2012, 235, 779-791. [CrossRef] [PubMed]

20. Feng, Z.-J.; He, G.-H.; Zheng, W.-J.; Lu, P.-P.; Chen, M.; Gong, Y.; Ma, Y.-Z.; Xu, Z.-S. Foxtail millet NF-Y families: Genome-wide survey and evolution analyses identified two functional genes important in abiotic stresses. Front. Plant Sci. 2015, 6, 1142. [CrossRef] [PubMed]

21. Mei, X.; Liu, C.; Yu, T.; Liu, X.; Xu, D.; Wang, J.; Wang, G.; Cai, Y. Identification and characterization of paternal-preferentially expressed gene NF-YC8 in maize endosperm. Mol. Genet. Genom. 2015, 290, 1819-1831. [CrossRef] [PubMed]

22. Zhang, Z.; Li, X.; Zhang, C.; Zou, H.; Wu, Z. Isolation, structural analysis, and expression characteristics of the maize nuclear factor Y gene families. Biochem. Biophys. Res. Commun. 2016, 478, 752-758. [CrossRef] [PubMed]

23. Malviya, N.; Jaiswal, P.; Yadav, D. Genome-wide characterization of Nuclear Factor Y (NF-Y) gene family of sorghum [Sorghum bicolor (L.) Moench]: A bioinformatics approach. Physiol. Mol. Biol. Plants 2016, 22, $33-49$. [CrossRef] [PubMed]

24. Gusmaroli, G.; Tonelli, C.; Mantovani, R. Regulation of the CCAAT-binding NF-Y subunits in Arabidopsis thaliana. Gene 2001, 264, 173-185. [CrossRef]

25. Siefers, N.; Dang, K.K.; Kumimoto, R.W.; Bynum, W.E.T.; Tayrose, G.; Holt, B.F., 3rd. Tissue-specific expression patterns of Arabidopsis NF-Y transcription factors suggest potential for extensive combinatorial complexity. Plant Physiol. 2009, 149, 625-641. [CrossRef] [PubMed]

26. Ripodas, C.; Castaingts, M.; Clua, J.; Blanco, F.; Zanetti, M.E. Annotation, phylogeny and expression analysis of the nuclear factor $\mathrm{Y}$ gene families in common bean (Phaseolus vulgaris). Front. Plant Sci. 2014, 5, 761. [CrossRef] [PubMed]

27. Liang, M.; Yin, X.; Lin, Z.; Zheng, Q.; Liu, G.; Zhao, G. Identification and characterization of NF-Y transcription factor families in canola (Brassica napus L.). Planta 2014, 239, 107-126. [CrossRef] [PubMed]

28. Xu, L.; Lin, Z.; Tao, Q.; Liang, M.; Zhao, G.; Yin, X.; Fu, R. Multiple NUCLEAR FACTOR Y transcription factors respond to abiotic stress in Brassica napus L. PLoS ONE 2014, 9, e111354. [CrossRef] [PubMed]

29. Quach, T.N.; Nguyen, H.T.; Valliyodan, B.; Joshi, T.; Xu, D.; Nguyen, H.T. Genome-wide expression analysis of soybean NF-Y genes reveals potential function in development and drought response. Mol. Genet. Genom. 2015, 290, 1095-1115. [CrossRef] [PubMed]

30. Ren, C.; Zhang, Z.; Wang, Y.; Li, S.; Liang, Z. Genome-wide identification and characterization of the NF- $Y$ gene family in grape (Vitis vinifera L.). BMC Genom. 2016, 17, 605. [CrossRef] [PubMed]

31. Li, S.; Li, K.; Ju, Z.; Cao, D.; Fu, D.; Zhu, H.; Zhu, B.; Luo, Y. Genome-wide analysis of tomato NF-Y factors and their role in fruit ripening. BMC Genom. 2016, 17, 36. [CrossRef] [PubMed]

32. Chen, Y.; Yang, Z.; Xiao, Y.; Wang, P.; Wang, Y.; Ge, X.; Zhang, C.; Zhang, X.; Li, F. Genome-wide analysis of the NF-YB gene family in Gossypium hirsutum L. and characterization of the role of GhDNF-YB22 in embryogenesis. Int. J. Mol. Sci. 2018, 19, 483. [CrossRef] [PubMed]

33. Pereira, S.L.S.; Martins, C.P.S.; Sousa, A.O.; Camillo, L.R.; Araujo, C.P.; Alcantara, G.M.; Camargo, D.S.; Cidade, L.C.; de Almeida, A.F.; Costa, M.G.C. Genome-wide characterization and expression analysis of citrus NUCLEAR FACTOR-Y (NF-Y) transcription factors identified a novel NF-YA gene involved in drought-stress response and tolerance. PLOS ONE 2018, 13, e0199187. [CrossRef] [PubMed]

34. Wang, Y.; Xu, W.; Chen, Z.; Han, B.; Haque, M.E.; Liu, A. Gene structure, expression pattern and interaction of Nuclear Factor-Y family in castor bean (Ricinus communis). Planta 2018, 247, 559-572. [CrossRef] [PubMed]

35. Varshney, R.K.; Song, C.; Saxena, R.K.; Azam, S.; Yu, S.; Sharpe, A.G.; Cannon, S.; Baek, J.; Rosen, B.D.; Tar'an, B.; et al. Draft genome sequence of chickpea (Cicer arietinum) provides a resource for trait improvement. Nat. Biotechnol. 2013, 31, 240-246. [CrossRef] [PubMed]

36. Jain, M.; Misra, G.; Patel, R.K.; Priya, P.; Jhanwar, S.; Khan, A.W.; Shah, N.; Singh, V.K.; Garg, R.; Jeena, G.; et al. A draft genome sequence of the pulse crop chickpea (Cicer arietinum L.). Plant J. 2013, 74, 715-729. [CrossRef] [PubMed] 
37. Zheng, Y.; Jiao, C.; Sun, H.; Rosli, H.G.; Pombo, M.A.; Zhang, P.; Banf, M.; Dai, X.; Martin, G.B.; Giovannoni, J.J.; et al. iTAK: A program for genome-wide prediction and classification of plant transcription factors, transcriptional regulators, and protein kinases. Mol. Plant 2016, 9, 1667-1670. [CrossRef] [PubMed]

38. Jin, J.; Tian, F.; Yang, D.-C.; Meng, Y.-Q.; Kong, L.; Luo, J.; Gao, G. PlantTFDB 4.0: Toward a central hub for transcription factors and regulatory interactions in plants. Nucleic Acids Res. 2017, 45, D1040-D1045. [CrossRef] [PubMed]

39. Larkin, M.A.; Blackshields, G.; Brown, N.P.; Chenna, R.; McGettigan, P.A.; McWilliam, H.; Valentin, F.; Wallace, I.M.; Wilm, A.; Lopez, R.; et al. Clustal W and Clustal X version 2.0. Bioinformatics 2007, 23, 2947-2948. [CrossRef] [PubMed]

40. Hu, B.; Jin, J.; Guo, A.Y.; Zhang, H.; Luo, J.; Gao, G. GSDS 2.0: An upgraded gene feature visualization server. Bioinformatics 2015, 31, 1296-1297. [CrossRef] [PubMed]

41. Fedorova, L.; Fedorov, A. Introns in gene evolution. Genetica 2003, 118, 123-131. [CrossRef] [PubMed]

42. Le Hir, H.; Nott, A.; Moore, M.J. How introns influence and enhance eukaryotic gene expression. Trends Biochem. Sci. 2003, 28, 215-220. [CrossRef]

43. Roy, S.W.; Gilbert, W. The evolution of spliceosomal introns: Patterns, puzzles and progress. Nat. Rev. Genet. 2006, 7, 211-221. [PubMed]

44. Gasteiger, E.; Gattiker, A.; Hoogland, C.; Ivanyi, I.; Appel, R.D.; Bairoch, A. ExPASy: The proteomics server for in-depth protein knowledge and analysis. Nucleic Acids Res. 2003, 31, 3784-3788. [CrossRef] [PubMed]

45. Guruprasad, K.; Reddy, B.V.; Pandit, M.W. Correlation between stability of a protein and its dipeptide composition: A novel approach for predicting in vivo stability of a protein from its primary sequence. Protein Eng. 1990, 4, 155-161. [CrossRef] [PubMed]

46. Kyte, J.; Doolittle, R.F. A simple method for displaying the hydropathic character of a protein. J. Mol. Biol. 1982, 157, 105-132. [CrossRef]

47. Mantovani, R. The molecular biology of the CCAAT-binding factor NF-Y. Gene 1999, 239, 15-27. [CrossRef]

48. Hall, T.A. BioEdit: A user-friendly biological sequence alignment editor and analysis program for Windows 95/98/NT. Nucleic Acids Symp. Ser. 1999, 41, 95-98.

49. Cannon, S.; Mitra, A.; Baumgarten, A.; Young, N.; May, G. The roles of segmental and tandem gene duplication in the evolution of large gene families in Arabidopsis thaliana. BMC Plant Biol. 2004, 4, 10. [CrossRef] [PubMed]

50. Kawahara, Y.; De la Bastide, M.; Hamilton, J.P.; Kanamori, H.; McCombie, W.R.; Ouyang, S.; Schwartz, D.C.; Tanaka, T.; Wu, J.; Zhou, S.; et al. Improvement of the Oryza sativa nipponbare reference genome using next generation sequence and optical map data. Rice 2013, 6, 4. [CrossRef] [PubMed]

51. Librado, P.; Rozas, J. DnaSP v5: A software for comprehensive analysis of DNA polymorphism data. Bioinformatics 2009, 25, 1451-1452. [CrossRef] [PubMed]

52. Li, J.; Zhang, Z.; Vang, S.; Yu, J.; Wong, G.K.-S.; Wang, J. Correlation between Ka/Ks and Ks is related to substitution model and evolutionary lineage. J. Mol. Evol. 2009, 68, 414-423. [CrossRef] [PubMed]

53. Guo, Y.; Qiu, L.-J. Genome-wide analysis of the Dof transcription factor gene family reveals soybean-specific duplicable and functional characteristics. PLoS ONE 2013, 8, e76809. [CrossRef] [PubMed]

54. Gabaldón, T. Evolution of proteins and proteomes: A phylogenetics approach. Evol. Bioinform. 2005, 1, 51-61. [CrossRef]

55. Sulieman, S.; Van Ha, C.; Nasr Esfahani, M.; Watanabe, Y.; Nishiyama, R.; Pham, C.T.; Van Nguyen, D.; Tran, L.S. DT2008: A promising new genetic resource for improved drought tolerance in soybean when solely dependent on symbiotic $\mathrm{N}_{2}$ fixation. Biomed. Res. Int. 2015, 2015, 687213. [CrossRef] [PubMed]

56. Li, W.; Mallano, A.I.; Bo, L.; Wang, T.; Nisa, Z.; Li, Y. Soybean transcription factor GmNF-YB1 confers abiotic stress tolerance to transgenic Arabidopsis plants. Can. J. Plant Sci. 2016, 97, 501-515.

57. Esfahani, M.N.; Sulieman, S.; Schulze, J.; Yamaguchi-Shinozaki, K.; Shinozaki, K.; Tran, L.S. Mechanisms of physiological adjustment of $\mathrm{N}_{2}$ fixation in Cicer arietinum L. (chickpea) during early stages of water deficit: single or multi-factor controls. Plant J. 2014, 79, 964-980. [CrossRef] [PubMed]

58. Mochida, K.; Yoshida, T.; Sakurai, T.; Yamaguchi-Shinozaki, K.; Shinozaki, K.; Tran, L.S. In silico analysis of transcription factor repertoires and prediction of stress-responsive transcription factors from six major gramineae plants. DNA Res. 2011, 18, 321-332. [CrossRef] [PubMed] 
59. Zhang, W.; Ruan, J.; Ho, T.H.; You, Y.; Yu, T.; Quatrano, R.S. Cis-regulatory element based targeted gene finding: Genome-wide identification of abscisic acid- and abiotic stress-responsive genes in Arabidopsis thaliana. Bioinformatics 2005, 21, 3074-3081. [CrossRef] [PubMed]

60. Nakashima, K.; Yamaguchi-Shinozaki, K. ABA signaling in stress-response and seed development. Plant Cell. Rep. 2013, 32, 959-970. [CrossRef] [PubMed]

61. Mathiyalagan, R.; Muthurajan, R.; Subramaniyam, S.; Jegadeesan, R. In silico analysis of drought tolerant genes in rice. Int. J. Biol. Med. Res. 2010, 1, 36-40.

62. Maruyama, K.; Todaka, D.; Mizoi, J.; Yoshida, T.; Kidokoro, S.; Matsukura, S.; Takasaki, H.; Sakurai, T.; Yamamoto, Y.Y.; Yoshiwara, K.; et al. Identification of cis-acting promoter elements in cold- and dehydration-induced transcriptional pathways in Arabidopsis, rice, and soybean. DNA Res. 2012, 19, 37-49. [CrossRef] [PubMed]

63. Abe, H.; Urao, T.; Ito, T.; Seki, M.; Shinozaki, K.; Yamaguchi-Shinozaki, K. Arabidopsis AtMYC2 (bHLH) and AtMYB2 (MYB) function as transcriptional activators in abscisic acid signaling. Plant Cell 2003, 15, 63-78. [CrossRef] [PubMed]

64. Yamaguchi-Shinozaki, K.; Shinozaki, K. Organization of cis-acting regulatory elements in osmotic- and cold-stress-responsive promoters. Trends Plant Sci. 2005, 10, 88-94. [CrossRef] [PubMed]

65. Dubouzet, J.G.; Sakuma, Y.; Ito, Y.; Kasuga, M.; Dubouzet, E.G.; Miura, S.; Seki, M.; Shinozaki, K.; Yamaguchi-Shinozaki, K. OsDREB genes in rice, Oryza sativa L., encode transcription activators that function in drought-, high-salt- and cold-responsive gene expression. Plant J. 2003, 33, 751-763. [CrossRef] [PubMed]

66. Tran, L.S.; Nakashima, K.; Sakuma, Y.; Simpson, S.D.; Fujita, Y.; Maruyama, K.; Fujita, M.; Seki, M.; Shinozaki, K.; Yamaguchi-Shinozaki, K. Isolation and functional analysis of Arabidopsis stress-inducible NAC transcription factors that bind to a drought-responsive cis-element in the early responsive to dehydration stress 1 promoter. Plant Cell 2004, 16, 2481-2498. [CrossRef] [PubMed]

67. Nakashima, K.; Tran, L.S.; Nguyen, D.; Fujita, M.; Maruyama, K.; Todaka, D.; Ito, Y.; Hayashi, N.; Shinozaki, K.; Yamaguchi-Shinozaki, K. Functional analysis of a NAC-type transcription factor OsNAC6 involved in abiotic and biotic stress-responsive gene expression in rice. Plant J. 2007, 51, 617-630. [CrossRef] [PubMed]

68. Nguyen, K.H.; Mostofa, M.G.; Li, W.; Van Ha, C.; Watanabe, Y.; Le, D.T.; Thao, N.P.; Tran, L.-S.P. The soybean transcription factor GmNAC085 enhances drought tolerance in Arabidopsis. Environ. Exp. Bot. 2018, 151, 12-20. [CrossRef]

69. Chen, M.; Wang, Q.Y.; Cheng, X.G.; Xu, Z.S.; Li, L.C.; Ye, X.G.; Xia, L.Q.; Ma, Y.Z. GmDREB2, a soybean DRE-binding transcription factor, conferred drought and high-salt tolerance in transgenic plants. Biochem. Biophys. Res. Commun. 2007, 353, 299-305. [CrossRef] [PubMed]

70. Hussain, R.M.; Ali, M.; Feng, X.; Li, X. The essence of NAC gene family to the cultivation of drought-resistant soybean (Glycine max L. Merr.) cultivars. BMC Plant Biol. 2017, 17, 55. [CrossRef] [PubMed]

71. Pontes, B.; Giráldez, R.; Aguilar-Ruiz, J.S. Configurable pattern-based evolutionary biclustering of gene expression data. Algorithms Mol. Biol. 2013, 8, 4. [CrossRef] [PubMed]

72. Verma, M.; Kumar, V.; Patel, R.K.; Garg, R.; Jain, M. CTDB: An integrated chickpea transcriptome database for functional and applied genomics. PLoS ONE 2015, 10, e0136880. [CrossRef] [PubMed]

73. Garg, R.; Patel, R.K.; Jhanwar, S.; Priya, P.; Bhattacharjee, A.; Yadav, G.; Bhatia, S.; Chattopadhyay, D.; Tyagi, A.K.; Jain, M. Gene discovery and tissue-specific transcriptome analysis in chickpea with massively parallel pyrosequencing and web resource development. Plant Physiol. 2011, 156, 1661-1678. [CrossRef] [PubMed]

74. Garg, R.; Sahoo, A.; Tyagi, A.K.; Jain, M. Validation of internal control genes for quantitative gene expression studies in chickpea (Cicer arietinum L.). Biochem. Biophys. Res. Commun. 2010, 396, 283-288. [CrossRef] [PubMed]

75. Kumimoto, R.W.; Zhang, Y.; Siefers, N.; Holt, B.F., 3rd. NF-YC3, NF-YC4 and NF-YC9 are required for CONSTANS-mediated, photoperiod-dependent flowering in Arabidopsis thaliana. Plant J. 2010, 63, 379-391. [CrossRef] [PubMed]

76. Hwang, Y.H.; Kim, S.K.; Lee, K.C.; Chung, Y.S.; Lee, J.H.; Kim, J.K. Functional conservation of rice OsNF-YB/YC and Arabidopsis AtNF-YB/YC proteins in the regulation of flowering time. Plant Cell Rep. 2016, 35, 857-865. [CrossRef] [PubMed] 
77. O'Connor, L.; Gilmour, J.; Bonifer, C. The role of the ubiquitously expressed transcription factor Sp1 in tissue-specific transcriptional regulation and in disease. Yale J. Biol. Med. 2016, 89, 513-525. [PubMed]

78. Nelson, D.E.; Repetti, P.P.; Adams, T.R.; Creelman, R.A.; Wu, J.; Warner, D.C.; Anstrom, D.C.; Bensen, R.J.; Castiglioni, P.P.; Donnarummo, M.G.; et al. Plant nuclear factor Y (NF-Y) B subunits confer drought tolerance and lead to improved corn yields on water-limited acres. Proc. Natl. Acad. Sci. USA 2007, 104, 16450-16455. [CrossRef] [PubMed]

79. Zhang, T.; Zhang, D.; Liu, Y.; Luo, C.; Zhou, Y.; Zhang, L. Overexpression of a NF-YB3 transcription factor from Picea wilsonii confers tolerance to salinity and drought stress in transformed Arabidopsis thaliana. Plant Physiol. Biochem. 2015, 94, 153-164. [CrossRef] [PubMed]

80. Han, X.; Tang, S.; An, Y.; Zheng, D.C.; Xia, X.L.; Yin, W.L. Overexpression of the poplar NF-YB7 transcription factor confers drought tolerance and improves water-use efficiency in Arabidopsis. J. Exp. Bot. 2013, 64, 4589-4601. [CrossRef] [PubMed]

81. Chen, M.; Zhao, Y.; Zhuo, C.; Lu, S.; Guo, Z. Overexpression of a NF-YC transcription factor from bermudagrass confers tolerance to drought and salinity in transgenic rice. Plant Biotechnol. J. 2015, 13, 482-491. [CrossRef] [PubMed]

82. Lata, C.; Prasad, M. Role of DREBs in regulation of abiotic stress responses in plants. J. Exp. Bot. 2011, 62, 4731-4748. [CrossRef] [PubMed]

83. Vishwakarma, K.; Upadhyay, N.; Kumar, N.; Yadav, G.; Singh, J.; Mishra, R.K.; Kumar, V.; Verma, R.; Upadhyay, R.G.; Pandey, M.; et al. Abscisic acid signaling and abiotic stress tolerance in plants: A review on current knowledge and future prospects. Front Plant Sci. 2017, 8, 161. [CrossRef] [PubMed]

84. Shinozaki, K.; Yamaguchi-Shinozaki, K. Gene networks involved in drought stress response and tolerance. J. Exp. Bot. 2007, 58, 221-227. [CrossRef] [PubMed]

85. Hurst, L.D. The Ka/Ks ratio: Diagnosing the form of sequence evolution. Trends Genet. 2002, 18, 486. [CrossRef]

86. Kumar, S.; Stecher, G.; Tamura, K. MEGA7: Molecular evolutionary genetics analysis version 7.0 for bigger datasets. Mol. Biol. Evol. 2016, 33, 1870-1874. [CrossRef] [PubMed]

87. Lescot, M.; Déhais, P.; Thijs, G.; Marchal, K.; Moreau, Y.; Van de Peer, Y.; Rouzé, P.; Rombauts, S. PlantCARE, a database of plant cis-acting regulatory elements and a portal to tools for in silico analysis of promoter sequences. Nucleic Acids Res. 2002, 30, 325-327. [CrossRef] [PubMed]

88. Le, D.T.; Nishiyama, R.; Watanabe, Y.; Mochida, K.; Yamaguchi-Shinozaki, K.; Shinozaki, K.; Tran, L.S. Genome-wide survey and expression analysis of the plant-specific NAC transcription factor family in soybean during development and dehydration stress. DNA Res. 2011, 18, 263-276. [CrossRef] [PubMed]

89. Rozen, S.; Skaletsky, H. Primer3 on the WWW for general users and for biologist programmers. Methods Mol. Biol. 2000, 132, 365-386. [PubMed]

(C) 2018 by the authors. Licensee MDPI, Basel, Switzerland. This article is an open access article distributed under the terms and conditions of the Creative Commons Attribution (CC BY) license (http:/ / creativecommons.org/licenses/by/4.0/). 